\title{
Radiation therapy and biologics: a ripe opportunity?
}

\author{
Joel E Tepper and Carolyn I Sartor
}

Intense efforts to produce therapeutic agents directed at cellular pathways dysregulated in cancer cells have been spectacularly successful-imatinib and trastuzumab being pertinent examples; however, many targeted therapeutics are not intrinsically cytotoxic, prompting the development of agents that can enhance the cell killing of established cytotoxic agents.

lonizing radiation is one of the most potent therapeutics, and the addition of chemotherapy to radiotherapy in those tumors has produced major advances. Radiation sensitization using biologics holds even more promise, yet combining such therapies has been relatively unexplored. Despite positive preclinical reports of radiosensitization of tumors by biologic agents, only one phase III trial of a biologic agent and radiation therapy has been performed, which resulted in an impressive improvement in local control and survival (Bonner JA et al. [2006] N Engl J Med 354: 567-578).

Why then is this approach not being studied more intensively? One reason is the complexity of such studies. The baseline response rates to radiotherapy are generally high, so detecting an increased response is difficult. Moreover, aggressive radiation regimens are primarily used in curative situations, where it is much harder to justify a trial of a new agent without strong preliminary data.

Importantly, most pharmaceutical companies seem reluctant to make radiosensitization the basis for drug development, which is possibly related to FDA approval strategies for new antineoplastic agents. Approval generally requires proof of clinical activity in the form of improved survival. While local control can significantly influence survival, in most situations it does not directly affect survival. Radiotherapy is typically employed only for palliation in patients with metastatic disease. Thus, new drugs are usually approved for use in combination with radiotherapy only after approval for systemic disease.
We need to

be able to

work more

effectively

with the

pharmaceutical

industry and

government

... our patients

deserve

nothing less.

JE Tepper is an Advisory Board member of Nature

Clinical Practice

Oncology. CI Sartor

is Co-Director of the

Breast Program at the

University of North

Carolina/Lineberger

Comprehensive

Cancer Center, USA.

Competing interests

JE Tepper declared he

has been a consultant

for Genentech and

Bristol-Myers-Squibb.

$\mathrm{Cl}$ Sartor has declared that

she receives clinical trial

support (drug only) from

GlaxoSmithKline.

www.nature.com/clinicalpractice doi:10.1038/ncponc0565
Promising radiosensitizers that are not particularly effective as single agents or when combined with chemotherapy may never make it to the post-approval phase of development. How many potential radiosensitizers are not being investigated further? FDA indications for approval are shifting with the changing expectations of biologic agents. Symptomatic improvement and time to progression are now recognized endpoints that lend themselves better to palliative radiotherapy settings. The pharmaceutical industry would be wise to expand their focus to radiotherapy-related applications, considering that the palliative radiotherapy market is expanding in this era of biologics.

Another underappreciated potential of biologic radiosensitizers is their ability to specifically target tumor processes, thereby reducing toxicity. Phase III studies that focus on biologic sensitizers versus cytotoxic sensitizers might not be an attractive pharmaceutical strategy but could benefit patients. One argument against this approach is the possible systemic control benefit from cytotoxics; however, when used as radiosensitizers, chemotherapy doses are often below those required for optimal systemic effect. Biologics can often be delivered at full dose with radiotherapy, and might retain systemic effects. Thus, trial designs that compare biologics with chemotherapeutics as radiosensitizers in locally advanced settings could be optimally suited to discern both local and systemic efficacy.

We need to develop strong preclinical data to justify specific clinical applications and then use model systems that can effectively test the concept in humans before phase III trials. Next, we need to design and implement clinical trials where proof of concept can be tested. Translational scientists and clinical researchers know many of the studies that need to be done. We need to be able to work more effectively with the pharmaceutical industry and government to assure that the proper studies are completed. Our patients deserve nothing less. 